\title{
O modelo de assistência à saúde mental das pessoas em uso problemático de drogas: uma reflexão sob a ótica dos Direitos Humanos dos Pacientes
}

The mental health care model of people with problematic drug use in Brazil: a reflection from the perspective of Patient Human Rights

El modelo de la atención a la salud mental de las personas con consumo problemático de drogas en Brasil: una reflexión desde la perspectiva de los Derechos Humanos de los Pacientes

Ângela Maria Rosas Cardoso ${ }^{1}$

Aline Albuquerque ${ }^{2}$

\begin{abstract}
Resumo
Objetivo: analisar o modelo de atenção em saúde mental fundamentado na Lei ํㅜ 10.216, de 2001 e as mudanças previstas na Lei no 13.840, de 2019, relacionadas ao tratamento de pessoas em uso problemático de drogas. Metodologia: pesquisa teórica e documental, baseada em literatura específica sobre as pessoas que fazem uso problemático de drogas, sustentada no arcabouço teórico-normativo dos Direitos Humanos dos Pacientes. Resultados: o Centro de Atenção Psicossocial (CAPS) é um dispositivo substitutivo à internação psiquiátrica, que promove a reabilitação psicossocial e a reinserção social dos pacientes. A Lei no 13.840 , de 2019, ao dispor sobre a internação involuntária de pessoas que fazem uso problemático de drogas e reforçar o modelo de abstinência, de institucionalização e das comunidades terapêuticas, mantém a lógica do cuidado centrado na estigmatização e no isolamento social, desrespeitando os princípios do cuidado centrado no paciente. Conclusão: as ações do governo mostram claramente que ainda há muitos caminhos de lutas e movimentações sociais para a garantia dos direitos adquiridos, visando a manutenção do tratamento digno ao paciente.
\end{abstract}

\section{Palavras-chave}

Direitos Humanos. Pacientes. Bioética. Transtornos relacionados ao uso de substâncias.

\begin{abstract}
Objective: to analyze the mental health care model based on the Law No. 10.216 of 2001 and the changes foreseen in the Law No. 13.840 of 2019, related to the treatment of people with problematic drug use. Methods: this is a theoretical and documentary research, based on specific literature on people in problematic drug use, supported by the theoreticalnormative framework of Patients' Human Rights. Results: the Psychosocial Care Center is a substitute device for psychiatric hospitalization, which promotes psychosocial rehabilitation and social reinsertion of patients. Thus, the Law No. 13.840 of 2019 , while dealing with the involuntary admission of people with problematic drug use and reinforces the model of abstinence, institutionalization and therapeutic communities, maintains the logic of care

\footnotetext{
${ }^{1}$ Doutoranda, Programa de Pós-Graduação em Bioética, Faculdade de Ciências da Saúde, Universidade de Brasília, Brasília, Distrito Federal, Brasil; enfermeira. https://orcid.org/0000-0002-3865-4320. E-mail: angelamrosas@gmail.com 2 Pós-doutora em Direitos Humanos, Universidade de Essex, Colchester, Essex, Reino Unido; professora, Programa de Pós-graduação em Bioética, Universidade de Brasília, Brasília, Distrito Federal, Brasil. https://orcid.org/0000-0002-55680790. E-mail: alineaoliveira@hotmail.com
} 
focused on stigmatization and social isolation, disregarding the principles of patient-centered care. Conclusion: The government's actions clearly show that there are still many paths of struggle and social movements to guarantee acquired rights, aiming at maintaining dignified treatment to the patient.

\section{Keywords}

Human Rights. Patients. Bioethics. Substance-related disorders.

\section{Resumen}

Objectivo: analizar el modelo de atención de salud mental, basado en la Ley N.․ 10.216/2001 y los cambios previstos en la Ley N.․ 13.840/2019, relacionados con el tratamiento de las personas con consumo problemático de drogas. Metodología: Investigación teórica y documental, basada en estudios de la literatura específica sobre las personas con consumo problemático de drogas, respaldado por el marco teórico-normativo de los Derechos Humanos de los Pacientes. Resultados: El Centro de Atención Psicosocial - CAPS- es un dispositivo sustituto de la internación psiquiátrica, que promueve la rehabilitación psicosocial y la reinserción social de los pacientes. La Ley N. 13.840 de 2019 al establecer la internación involuntaria de las personas con consumo problemático de drogas y reforzar el modelo de abstinencia, de institucionalización y de las comunidades terapêuticas, mantiene la lógica del cuidado centrada en la estigmatización y en el aislamiento social, no respetando los principios del cuidado, que tiene al paciente como figura central. Conclusión: Las acciones del gobierno muestran claramente que quedan muchos caminos de lucha y movilizaciones sociales para garantizar los derechos adquiridos, con la finalidad de mantener un tratamiento digno para el paciente.

\section{Palabras clave}

Derechos humanos. Los pacientes. Bioética. Trastornos relacionados con el uso de sustancias.

\section{Introdução}

A Lei ํㅜ 10.216, de 6 de abril de 2001, denominada de Lei da Reforma Psiquiátrica, estabeleceu a proteção e os direitos das pessoas com transtornos mentais e redirecionou o modelo de cuidado em saúde mental no Brasil (1). É considerada o marco legal da reforma psiquiátrica, e endossou, de forma histórica, as diretrizes da saúde mental na esfera do Sistema Único de Saúde (SUS), ao garantir às pessoas acometidas de transtornos mentais a universalidade de acesso aos serviços de saúde e o direito à assistência integral. Igualmente, a Lei da Reforma Psiquiátrica previu a descentralização do modelo de atendimento, ao determinar a estruturação de serviços mais próximos do convívio social de seus usuários, configurando redes assistenciais atentas às desigualdades existentes no Brasil (2). Esse redirecionamento objetivou o fortalecimento dos direitos humanos de pacientes, usuários e familiares ao propor modelos substitutivos à internação psiquiátrica, a partir da rede comunitária e territorial de serviços de saúde mental (1).

Com base na Lei oㅜ 10.216, de 2001, a Portaria oㅜ 336 do Ministério da Saúde (MS), de 19 de fevereiro de 2002, efetivou a reorientação do modelo de cuidado em saúde mental, 
priorizando a reabilitação psicossocial e a reinserção social das pessoas com transtornos mentais e das pessoas que fazem uso problemático de álcool e outras drogas. Tal efetivação ocorreu ao se instituir os Centros de Atenção Psicossocial (CAPS) como componentes do atendimento aos pacientes com transtornos mentais severos e persistentes em sua área territorial, em regime de tratamento intensivo, semi-intensivo e não intensivo (3). Ainda, foram demarcadas as distintas modalidades de CAPS, dentre as quais, os serviços destinados às pessoas que fazem uso problemático de álcool e outras drogas $(3,4)$.

A organização dos serviços de atenção em saúde mental como Rede de Atenção Psicossocial (RAPS) foi instituída com a publicação da Portaria no 3.088 , de 23 de dezembro de 2011, do Ministério da Saúde (MS), com os objetivos de ampliar o acesso à atenção psicossocial e de garantir a articulação de pontos de atenção à saúde, no âmbito do SUS (5). A criação da RAPS implementou o cuidado em saúde integral - com diferentes graus de complexidade -, possibilitando maior integração social, autonomia, protagonismo e participação das pessoas que fazem uso problemático de álcool e outras drogas. Salientase que a RAPS tem como diretriz o respeito aos Direitos Humanos, garantindo a autonomia e a liberdade das pessoas, estando em consonância com a Lei № 10.216, de 2001 (1, 5).

Especificamente, em relação às pessoas que fazem uso problemático de álcool e outras drogas, em 2004, foi criada a Política do Ministério da Saúde para a Atenção Integral a Usuários de Álcool e outras Drogas, na qual o Ministério da Saúde reconhece o atraso histórico na adoção de medidas acerca da temática pelo SUS, salientando que o consumo de álcool e outras drogas é um grave problema de saúde pública. A Política aborda a estratégia de redução de danos, mediante o desenvolvimento de ações de prevenção e cuidados prioritários às singularidades do paciente e o respeito à sua autonomia pessoal (6).

Em 2006, foi aprovada a Lei $n^{0} 11.343$, de 23 de agosto de 2006, que instituiu 0 Sistema Nacional de Políticas Públicas sobre Drogas (Sisnad) e prescreveu medidas para prevenção do uso indevido, atenção e reinserção social de pessoas que fazem uso problemático de tais substâncias (7). Registre-se que a Lei oํ 11.343, de 2006, foi alterada substantivamente pela Lei ㄲo 13.840, de 5 de junho de 2019, ao permitir a internação involuntária de usuários de drogas, reforçando o modelo de abstinência e das comunidades terapêuticas em detrimento da política de redução de danos e dos Centros de Atenção Psicossocial Álcool e Drogas (CAPS AD). Com o advento dessa lei, a internação poderá ser solicitada por familiar ou responsável legal, servidor público da área de saúde, de assistência 
social ou de órgãos públicos integrantes do SISNAD, sendo formalizada por decisão médica (8).

As alterações advindas da Lei no 13.840, de 2019, são consideradas retrocesso em relação às conquistas decorrentes da adoção do modelo de atenção em saúde mental preconizado pela Lei ํo 10.216, de 2001, que visa à construção de redes ampliadas e intersetoriais de atenção, de base comunitária, com ênfase na articulação entre serviços de diferentes níveis de complexidades e tendo como equipamento articulador central, o CAPS, apostando em ações humanizadoras na perspectiva de reabilitação psicossocial e redução do estigma e preconceito. As alterações foram objetos de crítica pela Organização das Nações Unidas (ONU) e estudiosos dos direitos humanos, que se ancoraram em diretrizes internacionais referentes ao tratamento de pessoas que fazem uso problemático de drogas, objetivando traçar caminhos para o enfrentamento dessa questão mediante afirmação da dignidade humana e do rechaço ao isolamento social (9).

A alteração legislativa contribui para políticas e práticas sociais contrárias aos direitos humanos das pessoas que fazem uso problemático de drogas (10) neste, por isso, este artigo parte do pressuposto de que as alterações efetuadas no modelo de atenção em saúde mental não se ajustam às diretrizes do SUS e da RAPS, que priorizam a atenção integral à pessoa em seu território, respeitando sua dignidade e autonomia.

No campo dos cuidados em saúde mental, a partir do fim do século $X X$, os Direitos Humanos têm-se constituído uma ferramenta essencial para salvaguardar a dignidade e a autonomia pessoal das pessoas com transtornos mentais e das pessoas que fazem uso problemático de drogas e para assegurar uma série de direitos específicos aplicados à esfera da saúde, tais como o direito à vida, à privacidade e à liberdade (11-13). Sua aplicação ao campo da saúde mental é essencial para evitar o retorno da institucionalização e a segregação de pessoas com transtornos mentais e daquelas que fazem uso problemático de drogas.

Peyraube (14) assinala que, em qualquer estratégia eleita para o tratamento de pessoas que fazem uso problemático de drogas, o respeito aos Direitos Humanos constitui um comando fundamental balizador do modelo de atenção à saúde mental. Ou seja, tal respeito se correlaciona com a prevenção de danos à saúde e à integridade pessoal, bem como da exclusão social do paciente.

Neste artigo, faz-se uso dos Direitos Humanos dos Pacientes (DHP), que constituem um referencial com arcabouço principiológico próprio e que contemplam as especificidades 
dos cuidados em saúde (15), composto pelos seguintes princípios: princípio do cuidado centrado no paciente; princípio da autonomia relacional; princípio da responsabilidade dos pacientes; e o princípio da dignidade humana (12). Este estudo enfatizará o princípio do cuidado centrado no paciente e tal escolha relaciona-se à importância dos seus elementos centrais - a dignidade e respeito, o compartilhamento de informação, participação e colaboração - na discussão do cuidado em saúde da pessoa que faz uso problemático de drogas no âmbito da RAPS. Registre-se que a aplicação dos direitos humanos no campo dos cuidados em saúde - particularmente ao paciente como sujeito de direito - encontra-se em construção, havendo escassos artigos e obras sobre a temática.

Este estudo objetiva analisar o modelo de atenção em saúde mental comunitário fundamentado na Lei n. .10 .216 , de 2001, considerando o CAPS como eixo organizador e substitutivo à internação psiquiátrica, contrapondo-se ao modelo de atenção à saúde preconizado pela Lei $n .-013.840$, de 2019 , com base no referencial dos DHP, precisamente no princípio do cuidado centrado no paciente.

\section{Metodologia}

Trata-se de pesquisa teórica e documental, baseada em estudos teóricos de literatura específica sobre a temática, bem como nos documentos produzidos pelo Relator Especial da ONU sobre Direito à Saúde (16) e nos Princípios para a Proteção das Pessoas com Transtornos Mentais e a Melhoria da Atenção à Saúde Mental, adotados pela Assembleia Geral da ONU por meio da Resolução n.․46/119, de 1991 (17), e da Legislação sobre Saúde Mental (18). Referente ao marco teórico, esta pesquisa sustenta-se no arcabouço teóriconormativo dos DHP, particularmente no princípio do cuidado centrado no paciente $(12,19$ 20). Quanto à escolha dos documentos assinalados, registra-se que o Relator Especial da ONU sobre Direito à Saúde é um especialista na temática, internacionalmente reconhecido, cujos relatórios são balizadores da atuação dos Estados, de organizações da sociedade civil e de entidades privada na esfera da saúde. Os Princípios para a Proteção das Pessoas com Transtornos Mentais e a Melhoria da Atenção à Saúde Mental são normas internacionais que norteiam as elaborações legislativas dos Estados, e a Legislação sobre Saúde Mental é uma produção da OMS que busca estabelecer parâmetros globais para a produção de leis em saúde mental.

Na primeira seção do texto, é contextualizada a construção da organização das políticas públicas na área de saúde mental no Brasil. Na segunda seção, é realizada uma 
contextualização do processo histórico da organização da política de saúde mental a partir da Lei no 10.216, de 2001, e dos documentos do Ministério da Saúde da Política de Atenção Integral aos Usuários de Álcool e Outras Drogas, em 2003, até a publicação da Lei oㅜ 13.840, de 2019. Na terceira seção, é exposto o referencial teórico dos DHP, tendo em vista os princípios e direitos humanos no contexto dos cuidados em saúde. E, por fim, a discussão sobre a relação entre os princípios do cuidado centrado no paciente e as normativas brasileiras relacionadas ao modelo de atenção em saúde mental comunitário fundamentado nos CAPS e na RAPS, considerando o CAPS como eixo organizador e substitutivo à internação psiquiátrica, contrapondo-se ao modelo de atenção à saúde preconizado pela Lei no 13.840 , de 2019 .

\section{O processo histórico da organização da construção do modelo de assistência à saúde mental no Brasil}

O modelo de assistência à saúde mental no Brasil é norteado pela reforma psiquiátrica e orientado pelo paradigma psicossocial. A partir dessa proposta, rompeu-se com o modelo manicomial de assistência à saúde mental, o qual tinha, como objeto do cuidado, a doença mental e adotava como estratégias de intervenção predominantes 0 isolamento e a resolutividade pela medicalização, cujo agente do cuidado é o médico e o lugar do cuidado, o hospital (21). Por outro turno, o paradigma psicossocial possui, como objeto de cuidado, a existência e o sofrimento da pessoa, sendo considerado um campo transdisciplinar com diversidades de intervenção, no qual há múltiplos profissionais e atores no cuidado (22). A reorganização da rede de atenção psicossocial se fundamenta em um modelo baseado no acesso universal e na promoção de direitos das pessoas, na convivência social e na articulação de ações e serviços de saúde em diferentes níveis de complexidade (23).

No desenho da RAPS, o CAPS é considerado o equipamento articulador central, no qual as necessidades e demandas dos usuários são norteadoras de ações territoriais que servem de suporte ao plano integrado de cuidados em saúde (5). Ainda, o CAPS tem como objetivo oferecer atendimento à população de sua área de abrangência, realizando o acompanhamento clínico e a reinserção social dos pacientes pelo acesso ao trabalho, lazer, exercício dos direitos e fortalecimento dos laços familiares e comunitários. É um serviço de atendimento de saúde mental criado para ser substitutivo às internações em hospitais psiquiátricos, destinado ao atendimento de pacientes com transtornos mentais severos e persistentes (23). 
A proposta do CAPS é a efetivação de práticas transformadoras no campo da saúde mental, a partir do entendimento de que é possível construir novas formas de se enfrentar o sofrimento psíquico. O CAPS parte da compreensão do paciente em sua complexidade, buscando assegurar seus direitos, rompendo com o isolamento, a exclusão e a estigmatização a que está submetido, quando a única possibilidade de enfrentamento é aquela ofertada pelo modelo psiquiátrico tradicional (24). A ruptura com o modelo manicomial significa muito mais que a busca pelo fim das internações psiquiátricas, pois aponta para uma construção profunda acerca do fenômeno do adoecimento mental, visando à reinserção social, ao resgate da autonomia, da cidadania e do reconhecimento das pessoas em sofrimento psíquico como sujeitos de direitos (25).

Entretanto, diante dessas diferenças paradigmáticas, há entraves para a implantação dos CAPS. Pante e Amarante ressaltam que "os mecanismos de institucionalização podem se dar mesmo em alguns serviços territoriais, ainda que estes tenham o objetivo de substituir o hospital psiquiátrico" (26, p.2075). Peres et al (27) apontam que para a efetivação do modelo psicossocial e da proposta do CAPS, há a necessidade da mobilização efetiva e da implicação dos diversos atores estatais nessa construção. Conforme as competências definidas em Lei, cabe ao Poder Executivo Federal formular e coordenar a execução da Política Nacional sobre Drogas, a fim de garantir a efetivação da política nos Estados e Munícipios. Entretanto, a despeito da política pública ser competência dos órgãos de saúde dos entes da federação, nos termos da repartição de competências constantes da Lei Orgânica da Saúde, destaca-se a competência dos Conselhos de Saúde para atuar na formulação de estratégias e no controle da execução da política de saúde.

A publicação Saúde Mental em Dados: 2003 - 2010, de 2015, descreve a expansão anual do CAPS desde o ano de 1998, mantendo uma taxa anual de crescimento relativamente estável, o que o Ministério da Saúde aponta como a consolidação desse modelo de atenção para pessoas com transtornos mentais e com necessidades decorrentes do uso de álcool e outras drogas, bem como para a ampliação de ofertas de cuidado de base territorial e comunitária (28). No entanto, os resultados de uma pesquisa documental, cujo objetivo foi identificar os avanços dos novos dispositivos implementados a partir da reforma psiquiátrica, indicaram que ainda existem importantes desafios para se ampliar a assistência ao paciente com transtorno mental, como: o aumento da cobertura dos CAPS; a implantação de leitos psiquiátricos em hospitais gerais; a integração da saúde mental com a atenção 
primária; e a desinstitucionalização de pessoas em situação de longa permanência hospitalar (29).

Novas discussões visando à reorganização da assistência para pessoas com sofrimento ou transtorno mental e com necessidades decorrentes do uso de crack, álcool e outras drogas, no âmbito do SUS, foram realizadas nas esferas legislativa e executiva sobre a Política de Saúde Mental, a partir da publicação da Resolução CIT № 32, de 14 de dezembro de 2017, da Comissão Intergestores Tripartite, instância de pactuação interfederativa dos aspectos operacionais da gestão do SUS (30), e da Portaria ํㅜ 3588 , de 21 de dezembro de 2017, do Ministério da Saúde (31). Nesses documentos, foram incluídos, a unidade de acolhimento (adulto e infanto-juvenil); enfermarias especializadas em hospital geral; hospital psiquiátrico; hospital-dia; comunidades terapêuticas; e o ambulatório multiprofissional de saúde mental como dispositivos de assistência na RAPS (31).

Entre outros dispositivos da RAPS citados no relatório Saúde Mental em Dados (28), está o aumento dos serviços residências terapêuticas (SRTs) ou comunidades terapêuticas, justificado diante das principais preocupações do processo de desinstitucionalização de pacientes psiquiátricos de longa permanência nos últimos anos. No entanto, apesar da proposta inicial das comunidades terapêuticas que "continham em si, a luta contra a hierarquização ou verticalidade dos papéis sociais, ou enfim, um processo de horizontalidade e democratização das relações", conforme apontado por Amarante (32), elas atualmente reproduzem o modelo de institucionalização psiquiátrica, o que tem sido amplamente criticado por estudiosos, profissionais e militantes da reforma psiquiátrica, conforme será discutido posteriormente.

$\mathrm{Em}$ face das novas normativas que representam significativo retrocesso diante das importantes conquistas oportunizadas pela reforma psiquiátrica, torna-se fundamental tratar da construção e da atualização da política pública concernente à assistência das pessoas em uso problemático de drogas.

\section{A construção e a atualização da Política Nacional sobre Drogas no Brasil}

As demandas relacionadas aos problemas decorrentes do uso de drogas pertencem ao campo de atenção à saúde mental, que vem passando por importantes transformações conceituais e operacionais. Pautada nos princípios do SUS, a atenção à saúde mental reorienta o modelo antes centrado na referência hospitalar para um novo modelo de atenção descentralizado e de base comunitária. O Ministério da Saúde lançou, em 2003, a Política 
de Atenção Integral aos Usuários de Álcool e Outras Drogas, que preconizou a estruturação e o fortalecimento de uma rede de assistência centrada na atenção comunitária e ênfase na reabilitação e reinserção social das pessoas que fazem uso problemático de álcool e outras drogas. Essa rede está alicerçada no CAPS AD, articulada à RAPS e ao restante da rede de saúde (2).

O III Levantamento Nacional sobre o Uso de Drogas Pela População Brasileira, realizado em 2017 pela Fundação Oswaldo Cruz e Secretaria Nacional de Políticas sobre Drogas, apontou que 3,3 milhões de pessoas maiores de 12 anos apresentaram critérios para dependência de álcool ou alguma substância psicoativa - exceto tabaco - nos 12 meses anteriores à pesquisa, representando $2,2 \%$ da população pesquisada, sendo que a maior prevalência se encontra na faixa etária de 25 a 34 anos (33). Em face desses aspectos, essas instituições ressaltam que o uso problemático de drogas se apresenta como um complexo problema de saúde pública, sendo necessários esforços nas ações de prevenção, tratamento e na reinserção da pessoa que faz uso problemático de álcool e outras drogas na sociedade (33).

A Lei n. -11.343 , de 2006, instituiu o SISNAD, cujos objetivos consistem em prescrever as medidas preventivas ao uso indevido, atenção e reinserção social das pessoas que fazem uso problemático de álcool e outras drogas, pois adota como premissa que essas pessoas deveriam ser assistidas pelas instituições de saúde e assistência social, a partir de ações de promoção e prevenção à saúde, a fim de reduzir os fatores de risco associados ao uso problemático e vulnerabilidade (7).

A partir da quebra de paradigmas no cuidado às pessoas que fazem uso problemático de álcool e outras drogas, houve a adoção da Política de Redução de Danos (PRD), constituindo um conjunto de estratégias de saúde pública voltadas para a minimização das consequências adversas causadas pelo uso abusivo de drogas (34). A pessoa que usa substâncias psicoativas, na perspectiva da PRD, é vista como ser ativo, capaz e útil para seus pares e a sociedade, sendo o protagonista de sua própria história de vida, e não relegado a um papel passivo. A pessoa é um sujeito de direitos, e não os deve ter suprimidos por fazer uso de substâncias psicoativas ilícitas (35).

O CAPS AD, como dispositivo da atenção psicossocial, é considerado substitutivo aos hospitais e leitos psiquiátricos, e nele os pacientes que fazem uso problemático de álcool e drogas encontram seu lugar na comunidade. É nesse ambiente que terão lugar as práticas 
terapêuticas, preventivas e educativas de maior impacto sobre os chamados fatores de risco para o uso problemático (2).

Perrone (36), ao analisar a trajetória da reforma psiquiátrica e do movimento de luta antimanicomial como marco de um processo de mudança na atenção em saúde mental, avalia que muitos hospitais psiquiátricos foram fechados, e, concomitantemente, foram abertas vagas em outros dispositivos visando à desinstitucionalização dos pacientes. No entanto, o problema da dependência do álcool e outras drogas tem se tornado cada vez mais alarmante, o que provoca, em função de ausência de políticas públicas consistentes e eficazes no campo do trabalho e da saúde, a proliferação de internação de pessoas principalmente em comunidades terapêuticas (CTs).

As CTs, como serviços de atenção em regime residencial, propostas na Portaria no 3.088, de 2011, e reafirmadas na Portaria ํㅜ 3.588, de 2017, ambas do Ministério da Saúde, foram idealizadas como equipamentos voltados à reinserção social dos pacientes e consideradas fundamentais para a desinstitucionalização de pacientes com história de longa permanência em hospitais psiquiátricos (31). Embora, em sua origem histórica, o movimento para implementação das CTs se aproxime do proposto pela reforma psiquiátrica e do movimento de luta antimanicomial, na prática, a realidade se mostrou diferente, como confirmado por diversos relatórios de inspeção (37). Com efeito, inspeções citadas no Relatório de Inspeção Nacional em Comunidades Terapêuticas - 2017, elaborado em conjunto pelo Conselho Federal de Psicologia, Mecanismo Nacional de Prevenção e Combate à Tortura, Procuradoria Federal dos Direitos do Cidadão e Ministério Público Federal e publicado em 2018 (37), apontam que uma boa parte das CTs no Brasil possui práticas tão desumanas e iatrogênicas quanto às das antigas instituições asilares manicomiais, sem garantir minimamente a preservação dos direitos humanos mais básicos. Por outro lado, Perrone (36) também ressalta que a grande maioria das CTs não recebe nenhuma forma de fiscalização, não se encontrando cadastrada em nenhum serviço de referência que regulamente sua prática, o que facilita ainda mais a proliferação de violações de direitos.

Outros problemas apontados por vários estudiosos quanto à proposta das CTs - tais como a inclusão das CTs no SUS e medidas relativas ao tratamento às pessoas que fazem uso problemático de drogas - têm levantado críticas e debates por parte de profissionais de saúde, conselhos de categorias profissionais e outras entidades interessadas. Esses atores sociais consideram que as propostas de financiamento das CTs substituem o tratamento 
médico por um programa terapêutico cuja eficácia não é comprovada cientificamente com recursos públicos, e impedem o investimento na ampliação da rede pública de saúde (37)(38)(39)(40).

Atualmente, as práticas utilizadas nas CTs remetem àquelas adotadas nos antigos asilos, colônias e reformatórios do início do século XX, que não apresentam registros de resultados positivos para os pacientes. Ademais, a história indica que esses modelos e práticas de saúde preconizam a segregação e a internação prolongada. Os problemas relacionados às CTs são ainda mais devastadores ao se analisar a Lei oㅜ 13.840, de 2019, que altera importantes aspectos da assistência às pessoas em uso problemático de drogas. Principalmente ao inserir o acolhimento em CTs como parte dos planos terapêuticos que visem à abstinência, mesmo diante das inúmeras fragilidades dessa modalidade apontadas nas inspeções realizadas por órgãos estatais.

Pita (39) sustenta que "se a vida é crônica, precisamos aprender a cuidar dos nossos usuários cronicamente, em liberdade, diuturnamente, oferecendo-Ihes chances de sobrevida digna nos diversos cenários em que a vida de todos os humanos se dá". Igualmente, enquadra as CTs como asilo-manicomiais que devem ser substituídas progressivamente, com ações efetivas que reforcem a consolidação do modelo de atenção à saúde mental proposto pela Lei oㅜ 10.216, de 2001.

É importante ressaltar que uma parte da população de pessoas em uso problemático de drogas vive em situação de risco social, sem acesso aos equipamentos sociais básicos necessários ao pleno desenvolvimento humano, além da falta de emprego e demais meios de geração de renda, acentuados níveis de pobreza e sem infraestrutura sanitária. É necessário ampliar cada vez mais o conjunto de ações de integração e apoio às populações vulneráveis e excluídas, que são marginalizadas, desassistidas e desinformadas de métodos de prevenção e das vias de acesso aos insumos de prevenção (41). Uma lei que reforça o uso da internação involuntária para tratamento de uso de drogas, atribuindo papel central às CTs dentro do plano terapêutico visando à abstinência, pode fortalecer uma prática higienista, atribuindo poder aos atores estatais para retirar pessoas em situação de rua, desrespeitando a sua autonomia pessoal e liberdade de locomoção.

As informações apresentadas no Relatório Mundial sobre Drogas 2018 ilustram a magnitude sem precedentes e a complexidade dos mercados globais de drogas. Consequências adversas para a saúde, causadas pelo consumo de drogas continuam a ser significativas, como as epidemias contínuas de opiáceos (42). Delgado (43) alerta que, 
apesar da gravidade da situação, não se pode usar o terror e o pânico gerados pela questão das drogas para legitimar a internação prolongada, própria da ultrapassada cultura manicomial.

Os esforços para apoiar a prevenção e o tratamento do uso de drogas também incluem fornecer às pessoas que fazem uso problemático de drogas o necessário conhecimento e habilidades para prevenir overdoses (42). Mas tais esforços apenas serão eficazes se forem baseados em evidências científicas e no respeito aos direitos humanos; e, ainda, na erradicação do estigma associado ao uso de drogas, a partir da compreensão de que os transtornos decorrentes do uso crônico de drogas e recidivas requerem intervenções multidisciplinares.

A nova Lei que orienta o tratamento às pessoas em uso problemático de uso de drogas no Brasil está na contramão das diretrizes mundiais, que reforçam a importância de ações de redução de danos. Ademais, os padrões internacionais preconizam ações que visem proporcionar e garantir o desenvolvimento e o retorno das pessoas à socialização com a inserção em atividades de trabalho, educação, lazer -, e que possam ampliar as perspectivas de vida, oferecendo melhores condições de desenvolvimento pessoal nos diferentes contextos nos quais estão inseridos.

Diante desses aspectos, o referencial dos Direitos Humanos dos Pacientes apresenta-se como um importante marco conceitual para subsidiar ações que visem a garantia dos direitos humanos dessas pessoas, em face do aumento das desigualdades e iniquidades sociais.

\section{Os Direitos Humanos dos Pacientes}

Os DHP consistem em um referencial normativo-teórico construído a partir de normativas de direitos humanos, aplicando-as ao contexto dos cuidados em saúde (12). Neste estudo, enfatiza-se os princípios do cuidado centrado no paciente e da dignidade humana, que são norteadores do fortalecimento das ações de saúde no cuidado ao paciente em uso problemático de drogas e a sua família. A efetiva aplicação dos princípios dos DHP concorre para o incremento da qualidade do cuidado, pois visam assegurar que o tratamento em saúde atenda às necessidades social, emocional e física do paciente, levando-se em conta seus valores e preferências (12).

No que tange ao princípio do cuidado centrado no paciente, destacam-se os pilares dignidade e respeito; compartilhamento de informação; participação; e colaboração - e seus 
componentes - a comunicação entre os profissionais de saúde e os pacientes; informação em relação ao processo de cuidado, procedimentos e efeitos colaterais; cultura organizacional que avalie as necessidades dos pacientes e estimule a sua participação e de seus familiares e amigos na elaboração; e acompanhamento do plano terapêutico (12).

O cuidado centrado no paciente considera o paciente como pessoa, e não como objeto da doença ou do cuidado; explora os sintomas apresentados por ela, considerando seus contextos de vida, bem como enfatiza a importância de se incluir a perspectiva de cada paciente a partir de uma visão biopsicossocial mais ampla. Assim, o cuidado centrado no paciente busca promover condições de agência a ele, e tomada de decisão compartilhada quanto ao tratamento (44).

O cuidado centrado no paciente também preconiza o estabelecimento de uma relação de confiança e respeito entre o paciente e o profissional de saúde, em que o profissional deve trabalhar o potencial humano e as competências de cada pessoa e a sua família (44), ao invés de enfocar nas deficiências ou fragilidades do paciente, o que é usual no cuidado em saúde de pessoas com transtornos mentais (19).

Freeth (19) reforça a necessidade de mudança dos profissionais quanto à confiança no potencial inato e na capacidade do paciente de superar e lidar com as suas dificuldades. Assim, o cuidado centrado no paciente assume que a adoção do suporte adequado às vulnerabilidades e ao seu adoecimento mental pode impactar positivamente em sua condição de saúde. Segundo o autor, esse tipo de abordagem visa aumentar o senso de liberdade e a responsabilidade das pessoas. Para tanto, o profissional deve assumir que os pacientes precisam de condições facilitadoras para exercer as suas capacidades.

\section{O modelo de atenção psicossocial no Brasil e os princípios do cuidado centrado no paciente}

Considerando os avanços e os retrocessos do modelo de atenção em psicossocial no Brasil e suas diretrizes para o funcionamento e a articulação dos dispositivos da rede de assistência psicossocial, tem-se como objetivo analisar o CAPS sob a ótica dos princípios do cuidado centrado no paciente e da dignidade humana, segundo os DHP. Sendo dispositivo da RAPS, o CAPS, nas suas diferentes modalidades, é considerado o principal instrumento de implementação da assistência à saúde mental, por isso, esse dispositivo deve ser entendido como uma estratégia de transformação da assistência que se concretiza em uma ampla rede de cuidados em saúde mental (45). 
O CAPS tem valor estratégico para a reforma psiquiátrica brasileira, porquanto sua atribuição é prestar atendimento clínico em regime de atenção diária, evitando, assim, as internações hospitalares. Para tal, busca promover a inserção social das pessoas com transtornos mentais e que fazem uso problemático de álcool e outras drogas por meio de ações intersetoriais; regular a porta de entrada da rede de assistência em saúde mental na sua área de atuação; e organizar a rede de atenção a pessoas com doenças mentais $(3,4)$.

O CAPS deve ser substitutivo e não complementar aos hospitais psiquiátricos, oferecendo atenção às pessoas com transtornos psíquicos graves e persistentes e que fazem uso problemático de álcool e outras drogas, procurando preservar e fortalecer os laços sociais do paciente em seu território. A presença da família de pacientes nos serviços de saúde mental está contemplada no CAPS, sendo considerada uma unidade cuidadora que possibilita o apoio e a busca de soluções para as dificuldades enfrentadas na vida cotidiana dos pacientes (45). O CAPS, ao incorporar a família no processo de cuidado do sujeito em sofrimento mental, se articula com o princípio do cuidado centrado no paciente, visto que nesse modelo é estabelecida uma parceria entre profissionais, pacientes e suas famílias.

Segundo o princípio do cuidado centrado no paciente, a família, considerada pelo paciente como tal, é compreendida como um elemento fundamental, sendo essencial para ele em alguns casos (12). Em estudo que analisou a participação da família no cuidado ao paciente no CAPS, identificou-se que a sua presença se constitui como um recurso que possibilita a construção de atividades de tratamento direcionadas ao paciente. Ademais, quando a família está presente, aumenta a oportunidade de esclarecer dúvidas, compreender o estado de saúde do paciente, receber orientações que auxiliam no enfrentamento de preconceitos, obter informações sobre o tipo de tratamento que o paciente receberá (46).

Os componentes do cuidado centrado no paciente, tais como a comunicação entre os profissionais de saúde e os pacientes, a informação em relação ao processo de cuidado, estímulo ao suporte afetivo de familiares e amigos, a participação do paciente na elaboração e acompanhamento do plano terapêutico, são encontrados na proposta de assistência do CAPS em suas diferentes modalidades.

Portanto, ao conferir ao paciente o direito de participar da construção de estratégias que incrementem sua saúde mental - com respeito à sua autonomia e dignidade -, esse tipo de cuidado também fortalece a relação entre o profissional e o paciente, reduzindo 0 abandono e o fracasso terapêutico (24). As alterações advindas com a Lei no 13.740, de 
2019 - notadamente quanto ao incentivo às internações psiquiátricas - impulsionam a adoção de ações de saúde focadas no adoecimento e na decisão quanto ao tratamento exclusivamente pelo médico e instituições de saúde.

Sublinha-se que o cuidado centrado no paciente tem como premissa a autonomia da pessoa e adota três dimensões: a primeira é que as pessoas têm o direito de tomar suas próprias decisões e realizar escolhas - mesmo que outras possam considerá-las irracionais -, também constituindo um princípio ético a ser adotado por todos os profissionais de saúde. A segunda, valoriza a capacidade de tomada de decisão do paciente, ressaltando que o objetivo principal de toda forma de cuidado deve ser promover a autonomia. A terceira dimensão diz respeito ao cuidado do paciente com transtorno mental, considerando os seus direitos e a capacidade de tomar as próprias decisões (19).

A implementação de ações de saúde visando ao cumprimento das diretrizes propostas na política de saúde mental devem ser norteadas pelo tratamento em saúde que atenda às necessidades social, emocional e física do paciente, considerando sua vontade $e$ preferências (12). O CAPS se apresenta como um importante dispositivo da RAPS para a efetivação dessa proposta. Portanto, a ampliação de CTs e o aumento do número de leitos em hospitais em oposição à falta de investimento na ampliação do CAPS caracterizam-se como um passo atrás no processo de construção de um modelo de atenção à saúde mental centrado no paciente e na promoção da sua dignidade.

Considerando a cronicidade do transtorno mental, também é importante ressaltar o papel do paciente no seu tratamento como um processo, pois o cuidado deve se basear na perspectiva do paciente para pensar no seu adoecimento e nos critérios de sucesso visando às intervenções, considerando que os cuidados devem possibilitar o aumento da capacidade do seu autocuidado. Assim, o tratamento bem-sucedido será definido não em termos de cessação da morbidade ou dos sintomas, mas na aquisição de competências e estratégias para conseguir alívio ao sofrimento e possibilitar a condução da vida de forma mais autônoma e como sujeito da sua história (20). Portanto, a internação involuntária como parte do tratamento do uso problemático de drogas não reconhece o paciente como sujeito de direitos ao desconsiderar sua capacidade de decisão e ao privá-lo de liberdade. Conforme o previsto na Lei oㅜ 13.840 , de 2019, ao dispor como alternativa de tratamento a internação involuntária apenas com o aval do profissional médico em unidades de saúde e hospitais gerais, tal diretriz reforça o cuidado centrado no adoecimento e nos processos históricos de exclusão social dessas pessoas, excluindo as demais áreas da saúde, a assistencial social 
e o paciente da construção do projeto terapêutico. Isto é, ela reforça a perspectiva médica do uso problemático de drogas centrada e reducionista, que visa apenas ao tratamento da patologia, apartando-se do contexto no qual as pessoas estão inseridas, bem como outras possibilidades de tratamento ou até mesmo de não tratamento.

Assim, presume-se que as pessoas em uso problemático de drogas são incapazes, pois, mesmo que não desejem se tratar e não sejam interditadas, o médico pode interná-las forçadamente. Segundo a Lei № 13.840, de 2019, a constatação da dependência abusiva do uso de drogas permite a internação - mesmo que não haja necessidade terapêutica -, apontando para a fragilidade do cuidado e o aumento de chance de tratamentos desumanos e degradantes de pessoas com transtorno mental.

Considerando que o SISNAD tem como princípio o respeito aos direitos humanos, especialmente quanto à sua autonomia e liberdade, observa-se clara ambiguidade entre os seus princípios e as propostas de efetivação da assistência aos pacientes em situação de uso problemático de álcool e outras drogas. Esses aspectos são apontados pelo Ministério Público Federal (47) ao destacar que a nova política desenhada pelo Ministério da Saúde apenas incentiva a manutenção de hospitais psiquiátricos e CTs. Ou seja, as novas diretrizes públicas negam às pessoas com transtorno mental o direito de serem cuidadas em serviços territorializados, sem o risco de segregação, que contribui para o agravo das condições de saúde das pessoas que fazem uso problemático de uso de drogas, violando a Lei oㅜ 10.216, de 2001. Portanto, esses aspectos ressaltados quanto às mudanças e aos retrocessos relativos ao tratamento das pessoas que fazem uso problemático de drogas se revelam em descompasso com os princípios do cuidado centrado no paciente.

\section{Considerações finais}

O princípio do cuidado centrado no paciente é norteador das ações de saúde ao paciente com transtorno mental e uso problemático de uso de drogas e sua família. Tais ações apontam para o cuidado singular, no qual o paciente participa do processo de construção do plano de tratamento, e atendem às pessoas em suas necessidades na perspectiva de proteção e de garantia de seus direitos humanos. A efetiva aplicação dos princípios dos Direitos Humanos do Paciente concorre para o incremento da qualidade do cuidado, pois visam assegurar que o tratamento em saúde atenda às necessidades social, emocional e física do paciente, levando em conta seus valores e preferências (12). 
Essas intervenções apontam claramente a intervenção do Estado na privação da liberdade, privacidade e autodeterminação, o que favorece condições de violação dos direitos humanos, principalmente quanto à dignidade humana. As ações do governo mostram claramente que ainda há muitos caminhos de lutas e movimentações sociais para a garantia dos direitos adquiridos, e do resgate em prol da manutenção do tratamento digno ao paciente com transtorno mental grave e persistente e uso problemático de uso de álcool e outras drogas.

\section{Referências}

1. Brasil. Lei no 10.216, de 6 de abril de 2001. Dispõe sobre a proteção e os direitos das pessoas portadores de transtornos mentais e redireciona o modelo assistencial em saúde mental. Diário Oficial da União [da] República Federativa do Brasil. (06 abr 2001), seção 1, 2.

2. Brasil. Ministério da Saúde. A Política do Ministério da Saúde para atenção integral a usuários de álcool e outras drogas. Brasília: Coordenação Nacional DST/AIDS; 2003.

3. Brasil. Ministério da Saúde. Portaria no 336, de 19 de fevereiro de 2002. Estabelece que os Centros de Atenção Psicossocial poderão constituir-se nas seguintes modalidades de serviços: CAPS I, CAPS II e CAPS III, definidos por ordem crescente de porte/complexidade e abrangência populacional. Diário Oficial da União [da] República Federativa do Brasil. (19 fev 2002). seção 1, 22.

4. Vargas AFM, Campos MM. A trajetória das políticas de saúde mental e de álcool e outras drogas no século XX. Ciência \& Saúde Coletiva. 2019;24(3):1041-1050.

5. Brasil. Ministério da Saúde. Portaria n 3.088, de 23 de dezembro de 2011. Instituiu a Rede de Atenção Psicossocial para pessoas com sofrimento ou transtorno mental e com necessidades decorrentes do uso de crack, álcool e outras drogas, no âmbito do Sistema Único de Saúde (SUS). Diário Oficial da União [da] República Federativa do Brasil. (23 dez 2011), seção 1.

6. Brasil. Ministério da Saúde. A Política do Ministério da Saúde para Atenção Integral a Usuários de Álcool e outras Drogas. 2ª .ed. Ministério da Saúde; 2004.

7. Brasil. Lei no 11.343, de 23 de agosto de 2006. Institui o Sistema Nacional de Políticas Públicas sobre Drogas - SISNAD. Diário oficial da União [da] República Federativa do Brasil. (23 ago 2006), seção 1.

8. Brasil. Lei no 13.840, de 5 de junho de 2019. Altera as Leis $n .-11.343$, de 23 de agosto de 2006, para dispor sobre o Sistema Nacional de Políticas Públicas sobre Drogas e as condições de atenção aos usuários ou dependentes de drogas e para tratar do financiamento das políticas sobre drogas. Diário Oficial da União [da] República Federativa do Brasil. (05 jun 2019). Seção 1, 1. 
9. Organização das Nações Unidas. ONU pede alternativas à prisão para quem sofre com dependência das drogas [Internet]. 26 jun 2019 [acesso em 01 jul 2019]. Disponível em: https://nacoesunidas.org/onu-pedealternativas-a-prisao-paraquemsofrecomdependenciadasdrogas/.

10. Costa PHA, Paiva FS. Revisão de literatura das concepções dos profissionais de saúde sobre o uso de drogas: modelo biomédico, naturalizações e moralismos. Physis. 2016;26(3):1009-1031.

11. Dhanda A, Narayan T. Mental health and human rights. The Lancet. 2007;370(9594):1197-1198.

12. Albuquerque A. Direitos humanos dos pacientes. Curitiba: Juruá; 2016.

13. Szmukler G, Bach M. Mental health disabilities and human rights protections. Global Mental Health. 2015;2(e20):1-9.

14. Peyraube R. Estigma de las personas que usan drogas, cuidados de la salud $y$ derechos humanos en américa latina. In: Dalla Vecchia $M$ et al, organizadores. Drogas e direitos humanos: reflexões em tempos de guerra às drogas [recurso eletrônico]. Porto Alegre: Rede UNIDA; 2017 [acesso em 02 dez 2020]. 396 p. Disponível em http://historico.redeunida.org.br/editora/biblioteca-digital/serie-interlocucoes-praticasexperiencias-e-pesquisas-em-saude/drogas-e-direitos-humanos-reflexoes-em-tempos-deguerra-as-drogas-pdf

15. Albuquerque A, Queiroz G, Paranhos D, Santos M. Os direitos humanos dos pacientes como novo referencial da bioética clínica. Revista Redbioética/Unesco. 2017;23(16):12-22.

16. Organização das Nações Unidas. Relator da ONU critica práticas de confinamento em nome da saúde pública [Internet]. 2018 [acesso em 17 nov 2019]. Disponível em:

https://nacoesunidas.org/relator-da-onu-critica-praticas-de-confinamento-nome-saudepublica/

17. CIDH. Principios para la protección de los enfermos mentales y el mejoramiento de la atención de la salud mental [Internet]. [s.d.] [acesso em 22 ago 2019]. Disponível em: https://www.cidh.oas.org/PRIVADAS/principiosproteccionmental.htm

18. Sullivan M. The Patient as Agent of Health and Health Care: Autonomy in patientcentered care for chronic conditions. Oxford University Press; 2017.

19. Organização Mundial da Saúde. La Importancia de los Derechos Humanos Consagrados Internacionalmente en la Legislación Nacional Sobre Salud Mental [Internet]. [s.d.] [acesso em 17 nov 2019]. Disponível em:

https://www.who.int/mental_health/policy/legislation/en/WHO_chapter_hr_spanish.pdf?ua= $1 \% 3 \mathrm{E}$

20. Freeth R. Humanising Psychiatry and Mental Health Care: the Challenge of the PersonCentred Approach. Oxford: Radcliffe; 2007. 
21. Sullivan M. The Patient as Agent of Health and Health Care. Oxford: Oxford University Press; 2017.

22. Tesser CD. Medicalização social e atenção à saúde no SUS. São Paulo: HUCITEC; 2010.

23. Costa-Rosa A. O modo Psicossocial: um paradigma das práticas substitutivas ao modo asilar. In: Amarante P (org.). Ensaios: subjetividade, saúde mental, sociedade. Rio de Janeiro: Fiocruz; 2000.

24. Brasil. Ministério da Saúde. Saúde mental no SUS: os centros de atenção psicossocial. Brasília: Departamento de Ações Programáticas Estratégicas; 2004.

25. Kantorski LP, Andrade APM. Assistência psiquiátrica mundo afora: práticas de resistência e garantia de direitos. Cadernos Brasileiros de Saúde Mental. 2017;9(24):50-72.

26. Lüchmann LHH, Rodrigues J. O movimento antimanicomial no Brasil. Ciência \& Saúde Coletiva. 2007;12(2):399-407.

27. Pande MNR, Amarante PDC. Desafios para os Centros de Atenção Psicossocial como serviços substitutivos: a nova cronicidade em questão. Ciência \& Saúde Coletiva. 2011;16(4):2067-2076.

28. Peres GM et al. Limites e desafios da rede de atenção psicossocial na perspectiva dos trabalhadores de saúde mental. Cadernos Brasileiros de Saúde Mental. 2018;10(27):34-52.

29. Brasil. Ministério da Saúde. Saúde Mental em Dados - 12 [Internet]. 2015; Ano 10, no

12. Disponível em: http://www.saude.gov.br/bvs/saudemental

30. Fagundes JR, Hugo M, Desviat M, Silva PRF. Reforma Psiquiátrica no Rio de Janeiro: situação atual e perspectivas futuras. Ciência \& Saúde Coletiva.2016;21(5):1449-1460.

31. Brasil. Ministério da Saúde. Resolução no 32, de 14 de dezembro de 2017. Diário Oficial da União [da] República Federativa do Brasil. (14 dez 2017). Seção 1, 239.

32. Brasil. Ministério da Saúde. Portaria $n^{\circ}$ 3588, de 21 de dezembro de 2017. Diário Oficial da União [da] República Federativa do Brasil. (21 dez 2017). Seção 1, 23-36.

33. Amarante P. Saúde mental e atenção psicossocial. Rio de Janeiro: Editora Fiocruz; 2007.

34. BASTOS FIPM et al, organizadores. III Levantamento Nacional sobre o Uso De Drogas Pela População Brasileira. Rio de Janeiro: ICICT/Fiocruz; 2017 [acesso em 10 nov 2019]. Disponível em: https://www.arca.fiocruz.br/handle/icict/34614]

35. Faria JG, Schneider DR. Relações entre racionalidade conservadora e pauperização do cuidado em CAPS AD: um estudo de caso. Cadernos Brasileiros de Saúde Mental. 2019;11(28):01-25. 
36. Passos EH, Souza TP. Redução de danos e saúde pública: construções alternativas à política global de "guerra às drogas". Psicologia \& Sociedade. 2011;23(1):154-162.

37. Perrone PAK. A comunidade terapêutica para recuperação da dependência do álcool e outras drogas no Brasil: mão ou contramão da reforma psiquiátrica?. Ciência \& Saúde Coletiva. 2014;19(2):569-580.

38. Conselho Federal de Psicologia. Relatório da Inspeção Nacional em Comunidades Terapêuticas - 2017.Brasília DF: CFP; 2018 [acesso em 10 nov 2019]. Disponível em: https://site.cfp.org.br/wp-content/uploads/2018/06/Relat\%C3\%B3rio-daInspe\%C3\%A7\%C3\%A3o-Nacional-em-Comunidades-Terap\%C3\%AAuticas.pdf

39. Bolonheis-Ramos RCM, Boarini ML. Comunidades terapêuticas: "novas" perspectivas e propostas higienistas. Rio de Janeiro: História, Ciências, Saúde-Manguinhos. 2015;22(4):1231-1248.

40. Pitta AMF. Um balanço da reforma psiquiátrica brasileira: instituições, atores e políticas. Ciência \&Saúde Coletiva. 2011; 16(12):4579-4589.

41. Amarante $P$, Nunes MO. A reforma psiquiátrica no SUS e a luta por uma sociedade sem manicômios. Ciência \& Saúde Coletiva. 2018;23(6):2067-74.

42. Büchele F, Magda DBD (Orgs). Curso de Atualização em Álcool e Outras Drogas, da Coerção à Coesão: recursos e estratégias do cuidado. Florianópolis: Departamento de Saúde Pública/UFSC; 2014 [acesso em 10 nov 2019] Disponível em: https://unasus.ufsc.br/alcooleoutrasdrogas/files/2015/03/M\%C3\%B3dulo-6.pdf

43. United Nations Office on Drugs and Crime (UNODC). World Drug Report 2018 [Internet]. United Nations; 2018 [acesso em 24 nov 2019]. Disponível em: https://www.unodc.org/wdr2018/prelaunch/WDR18_Booklet_1_EXSUM.pdf

44. Delgado PGG. Democracia e reforma psiquiátrica no Brasil. Ciência \& Saúde Coletiva. 2011;16(12):47014706.

45. Mead N, Bower P. Patient-centredness: a conceptual framework and review of the empirical literature. Social Science and Medicine. 2000;51:1087-1110.

46. Yasui S. Rupturas e encontros desafios da Reforma Psiquiátrica brasileira. Rio de Janeiro: Editora Fiocruz; 2010.

47. Moura MAP, Silva MGO, Morais TP. Centro de atenção psicossocial e a participação familiar no cuidado ao portador de sofrimento psíquico. Saúde Coletiva. 2013;10(60):19-24.

48. Procuradoria Federal dos Direitos do Cidadão. Nota pública PFDC acerca da proposta de alteração da política nacional de saúde mental [Internet]. 12 dez 2018 [acesso em 30 jun 2019]. Disponível em: http://pfdc.pgr.mpf.mp.br/temas-de-atuacao/saudemental/atuacaodompf/nota-publica-gtsaude-mental-pfdc-modificacaodediretrizesdaspoliticas-desaudemental-2017 


\section{Colaboradores}

Todos os autores contribuíram com a concepção, elaboração, redação, revisão e aprovação do artigo.

Como citar este artigo:

Cardoso AMR, Albuquerque A. O modelo de assistência à saúde mental das pessoas em uso problemático de drogas: uma reflexão sob a ótica dos Direitos Humanos dos Pacientes. Cadernos Ibero-Americanos de Direito Sanitário. 2020 out./dez.; 9(4): 135-155.

https://doi.org/10.17566/ciads.v9i4.611 